\title{
UTERINE LIPID METABOLISM IN MICE DURING THE OESTROUS CYCLE: EFFECT OF OVARIECTOMY AND REPLACEMENT THERAPY
}

\author{
AJIT GOSWAMI, AMIYA B. KAR AND S. R. GHOWDHURY \\ Central Drug Research Institute, Lucknow, India
}

(Received 22nd March 1963)

\begin{abstract}
Summary. Uterine lipid components of mice were analysed during different stages of the oestrous cycle and after ovariectomy and replacement therapy. During oestrus, and in ovariectomized animals subjected to oestrogen treatment, there was a fall in total lipid concentration and percentage of triglycerides, but an increase in concentration of phospholipids, as compared to the corresponding di-oestrous values. Progesterone antagonized the effect of oestrogen and restored the triglyceride concentration to the di-oestrous level. In comparison, the effect of progesterone on total lipids or phospholipids was less marked. There was no significant change in uterine cholesterol concentration during different stages of the oestrous cycle or after ovariectomy and hormone therapy. In general, the fatty acid components of the lipid constituents showed lower iodine values and increased saturation under the influence of oestrogen. These changes were more evident in triglyceride and phospholipid fractions than in the cholesterol ester fraction. Of the nitrogenous bases of phosphoglycerides, serine showed a more marked increase than the other components. The possible significance of these findings is discussed.
\end{abstract}

\section{INTRODUCTION}

Changes in distribution and concentration of stainable lipids in the uterus during different stages of the oestrous cycle were first reported in mice and subsequently extended to other species (Bourg, 1929; Gilbert, 1942; Alden, 1947; Page, Glendening \& Parkinson, 1951; Burgos \& Wislocki, 1956). However, except for the preliminary studies of Leathem (1959) and Davis \& Alden (1959), detailed quantitative chemical information on uterine lipid metabolism is meagre. The present investigation was therefore undertaken to study the details of uterine lipid metabolism in mice during the oestrous cycle and to elucidate the influence of ovarian hormones thereon.

\section{MATERIALS AND METHODS}

Colony-bred adult female albino mice ( 15 to $20 \mathrm{~g}$ ) of a Swiss strain maintained in the Institute were used in this investigation. For studies on uterine lipids 
animals which had shown regular cyclic oestrus for at least 2 months were selected by daily examination of their vaginal smears. All the animals were maintained under uniform laboratory conditions throughout the experimental period.

For study of hormonal effects, the mice were ovariectomized and subjected to therapy after a rest period of 2 weeks. Oestradiol-17- $\beta$ was injected subcutaneously at the rate of 1 and $5 \mu \mathrm{g}$ daily (in olive oil) for 4 days. Progesterone was administered similarly ( $5 \mathrm{mg}$ daily in olive oil) in combination with $1 \mathrm{\mu g}$ oestradiol-17- $\beta$ for 4 days. The control animals received the solvent alone by the subcutaneous route.

The animals were killed by decapitation. The uteri were carefully dissected out and weighed to the nearest $0.1 \mathrm{mg}$ in a Roller-Smith Balance after drying between pieces of filter paper. For dry weight determination, duplicate samples of the uteri were weighed similarly, dried at $110^{\circ} \mathrm{C}$ overnight and then weighed finally after cooling in a desiccator at room temperature.

The extraction and quantitative estimation of the total lipids, lipid components and the unsaturated fatty acids were by methods reported earlier (Goswami \& Sadhu, 1961).

For determination of nitrogenous bases of phospholipids, the latter were first isolated by the method of Van Beers, de Jongh \& Bolding (1958) and subsequently made up to suitable volumes with light petroleum ether. From aliquots, choline was estimated by the method of Wheeldon \& Collins (1958) and serine and ethanolamine by the procedure of Dittmer, Feminella \& Hanahan (1958).

\section{RESULTS}

UTERINE LIPID FRACTIONS IN DIFFERENT STAGES OF THE OESTROUS GYGLE

It will be seen from the data presented in Table 1 that during pro-oestrus and oestrus there was an increase in uterine water content which tended to lower the percentage dry weight (versus di-oestrus: $P<0 \cdot 01$ ). This trend continued even in metoestrus (versus di-oestrus: $P<0 \cdot 02$ ). The total lipid concentration showed a decrease during pro-oestrus and oestrus (versus di-oestrus: $P<0.05$ and $<0.01$, respectively). Concomitant with the reduction in total lipids, there was a significant decrease in the concentration of triglycerides (versus di-oestrus: $0.02>P>0.01)$. On the other hand, there was an overall increase in concentration of phospholipids and this was most marked during oestrus $(P<0.01)$. The concentration of uterine sterols did not register any significant change.

UTERINE LIPID FRACTIONS AFTER OVARIECTOMY AND AFTER REPLACEMENT THERAPY Uterine weight increased slightly but significantly after ovariectomy $(P<0.01$, Table 2). Oestrogen therapy either alone or in combination with progesterone to ovariectomized animals, reduced the uterine weight significantly (versus ovariectomized: $P<0 \cdot 01$ ). There seemed to be a dose effect in this respect as $5 \mu \mathrm{g}$ oestrogen was somewhat more effective than $1 \mu \mathrm{g}$. The percentage of total lipids increased after ovariectomy $(P<0.00)$ but declined after hormone therapy (versus ovariectomized: $P<0.01$ ) and tended to approach the normal value (Table 2 ). There was a similar increase in triglycerides after ovariectomy 
$(P<0.01)$ and reduction following oestrogen administration (versus ovariectomized: $P<0.01)$. However, progesterone antagonized the action of oestrogen on triglycerides (ovariectomized versus oestrogen plus progesterone: $P<0.4$ ). Ovariectomy had no statistically significant effect on esterified or free sterols of the uterus. But hormone therapy evoked different types of changes in the two fractions. Thus, $1 \mathrm{\mu g}$ oestrogen did not influence the percentage of esterified sterols in ovariectomized animals $(P<0 \cdot 1)$ but $5 \mu \mathrm{g}$ diminished it $(P<0 \cdot 01)$; combination of $1 \mu \mathrm{g}$ oestrogen with progesterone likewise reduced uterine sterol esters (versus ovariectomized: $P<0 \cdot 05$, Table 2 ). In contrast, free sterols were uniformly reduced after oestrogen therapy $(P<0 \cdot 05)$, whereas combined administration with progesterone did not alter the percentage of this fraction (Table 2). Ovariectomy lowered the percentage of phospholipids in the uterus $(P<0.01)$ but oestrogen treatment increased it consistently $(P<0 \cdot 01)$. However, the extent of this increase was significantly less in the combined oestrogen plus progesterone group $(P<0.01)$ suggesting some antagonism between the two hormones.

UTERINE FATTY ACID PATTERN AND PHOSPHOGLYGERIDE BASES DURING DIFFERENT STAGES OF THE OESTROUS CYCLE

The iodine value of polyenoic fatty acids of triglycerides was reduced slightly but significantly during pro-oestrus and oestrus (versus di-oestrus: $P<0 \cdot 05$, Table $3)$. No statistically significant change was, however, noticed during metoestrus. The only significant change that could be discerned in the fatty acid fractions was in dienoic and trienoic acids, but even this was confined to pro-oestrus and oestrus; there was a significant reduction in the percentage of these fractions (versus di-oestrus: $0.05>P>0.01$, Table 3 ).

There was a similar trend of reduction in iodine value of polyenoic fatty acids of sterol esters but except for pro-oestrus (versus di-oestrus: $P<0.01$, Table 4) none of them was significant statistically. Of the fatty acid fractions, only dienoic and tetraenoic showed any consistent change during oestrus; the former showed a decline (versus di-oestrus: $P<0.01$ ) and the latter an increase (versus di-oestrus: $P<0.01)$. The percentage of trienoic acid registered a significant reduction during pro-oestrus and oestrus (versus di-oestrus: $P<0.01$ ) but that of pentaenoic acid only during pro-oestrus (versus di-oestrus: $P<0.05$ ). The hexaenoic acid registered a slight but significant increase during pro-oestrus and oestrus (versus di-oestrus: $P<0 \cdot 01$ ) but not during metoestrus.

The iodine value of polyenoic fatty acids of phospholipids declined significantly during pro-oestrus and oestrus (versus di-oestrus: $P<0 \cdot 01$, Table 5 ) but remained virtually unchanged in metoestrus. The dienoic and trienoic fractions were not altered but the rest registered a consistent decline only during the pro-oestrus (versus di-oestrus: $0 \cdot 05>P>0 \cdot 01$ ). The penta- and hexaenoic fractions were reduced also during oestrus (versus di-oestrus: $P<0 \cdot 05$ ) but none showed a statistically significant change at metoestrus (Table 5).

Of the nitrogenous bases of phosphoglycerides, choline registered a significant decline at oestrus (versus di-oestrus: $P<0.05$, Table 6 ) but little change at other stages. The percentage of serine, on the other hand, increased significantly during pro-oestrus and oestrus (versus di-oestrus: $P<0.05$ ) but not in metoestrus. 

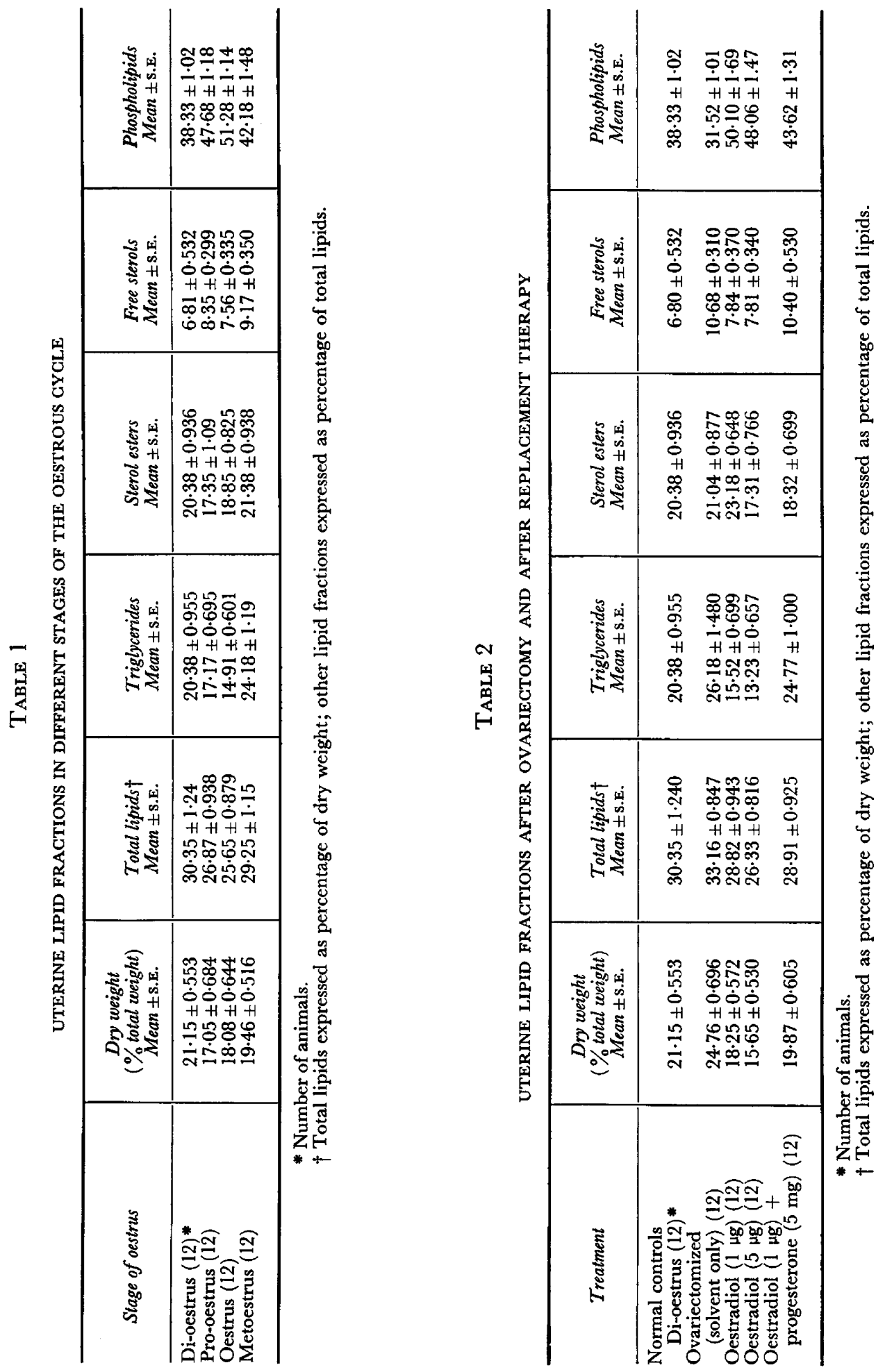


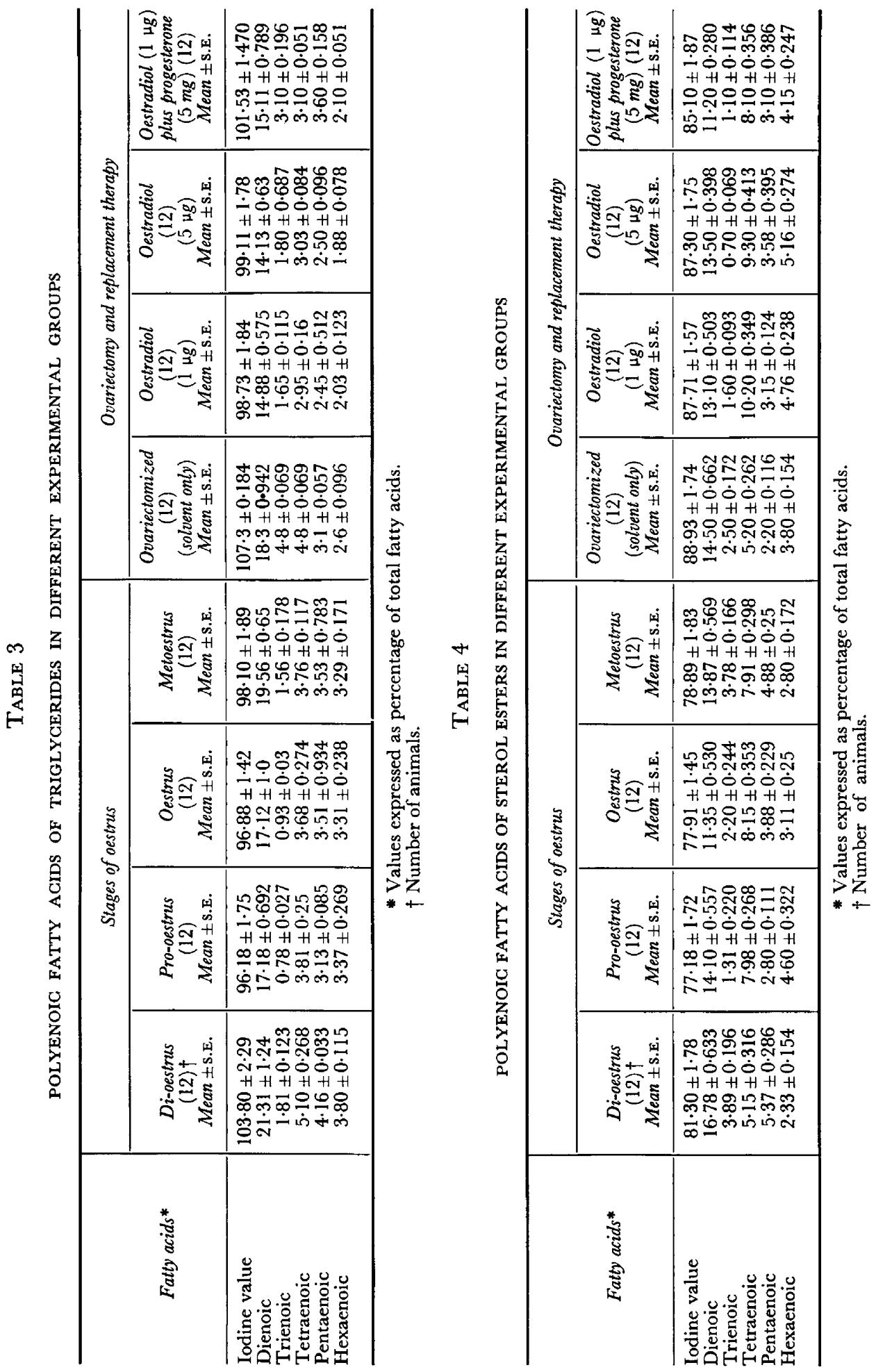



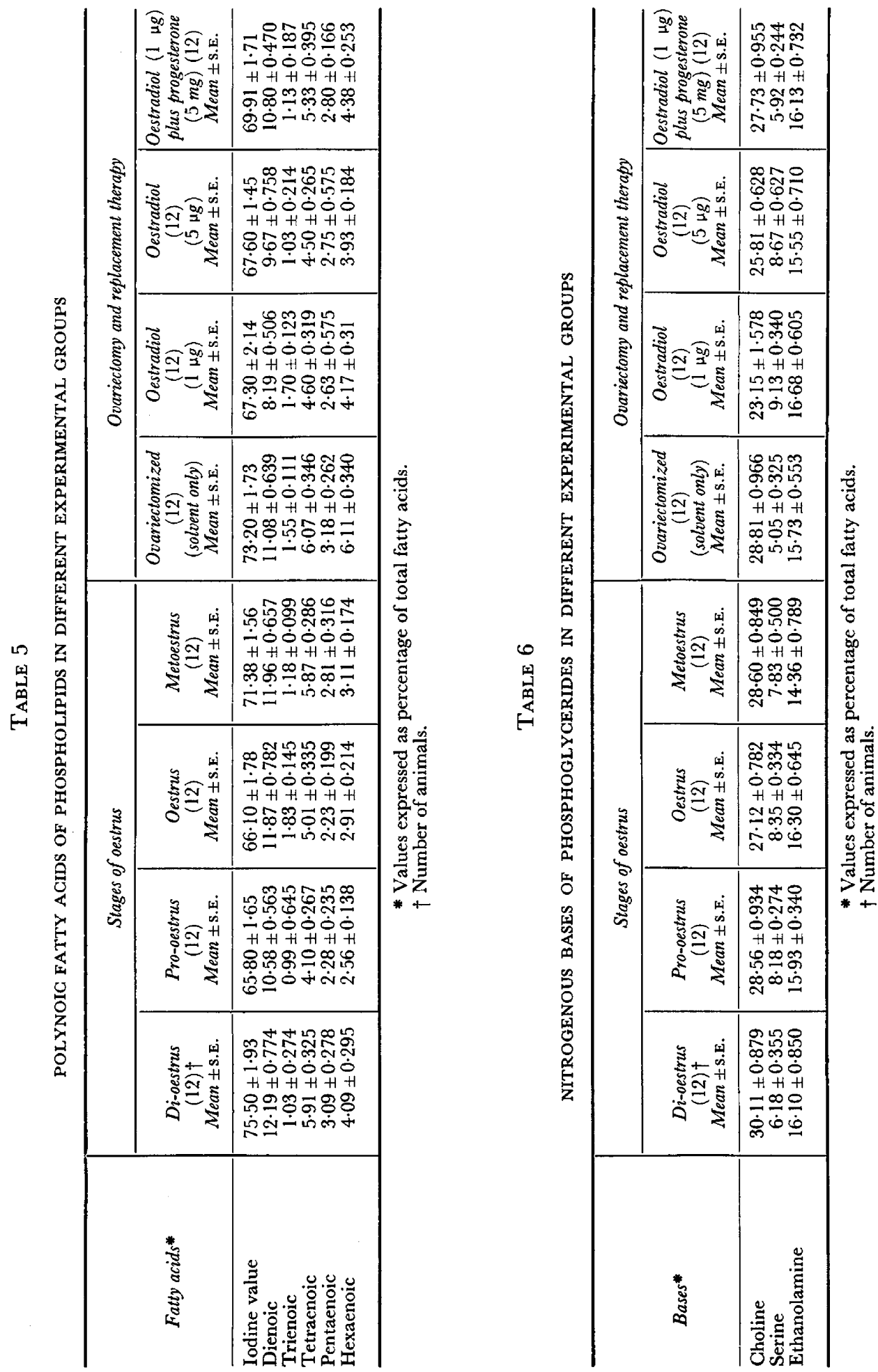
The oestrous cycle had virtually no effect on percentage of ethanolamine (Table 6).

UTERINE FATTY ACID PATTERN AND PHOSPHOGLYGERIDE BASES AFTER OVARIECTOMY AND AFTER REPLACEMENT THERAPY

The iodine value of polyenoic fatty acids of triglycerides did not alter significantly after ovariectomy (versus di-oestrus: $P<0 \cdot 2$, Table 3 ). However, the administration of hormones reduced the iodine values (versus ovariectomized: $P<0 \cdot 05$ ). Ovariectomy did not significantly alter the percentage of polyenoic fatty acids except for the trienoic fraction, which was elevated (versus di-oestrus $P<0 \cdot 01)$. Oestrogen therapy significantly lowered the dienoic acid percentage (versus ovariectomized: $0.05>P>0.01$, Table 3) but progesterone seemed to prevent it (ovariectomized versus oestrogen plus progesterone: $P<0 \cdot 1$ ). The trienoic fraction was consistently reduced after hormone therapy (versus ovariectomized: $0.05>P>0.01$ ), however, and progesterone prevented it (oestrogen $1 \mu \mathrm{g}$ versus oestrogen $1 \mu \mathrm{g}$ plus progesterone: $P<0 \cdot 01$, Table 3 ). Hormone administration had no significant effect on other fatty acid fractions.

Ovariectomy increased the iodine value of polyenoic fatty acid fractions of sterol esters (versus di-oestrus: $P<0 \cdot 01$, Table 4 ) but hormone therapy had no significant effect (versus ovariectomized: $P<0 \cdot 1$ ). The percentage of di-, tri- and pentaenoic fractions was reduced significantly after ovariectomy (versus dioestrus $0.05<P<0.01$ ) but that of the other acids remained virtually unchanged. The effect of hormone therapy on different fractions was varied. Thus, oestrogen per se did not significantly alter the percentage of dienoic acids but when administered in conjunction with progesterone tended to reduce it (versus ovariectomized: $P<0 \cdot 01$ ). The trienoic acids were, however, reduced consistently after hormone therapy (versus ovariectomized: $P<0.01$ ). On the other hand, the percentage of tetraenoic acids registered an increase after hormone treatment (versus ovariectomized: $P<0.01$ ). This was also the case with the pentaenoic fraction (versus ovariectomized: $P<0 \cdot 05$, Table 4 ). The hexaenoic fraction did not register any significant change after hormone therapy.

The iodine value of polyenoic fatty acids of phospholipids did not differ significantly after ovariectomy (versus di-oestrus: $P>0 \cdot 1$, Table 5) or after administration of oestrogen plus progesterone (versus ovariectomized: $P>0 \cdot 1$ ). However, oestrogen therapy (alone) reduced the iodine value significantly (versus ovariectomized: $P<0 \cdot 05)$. Ovariectomy increased the percentage of hexaenoic acids (versus di-oestrus: $P<0.05$ ) but had no significant effect on other fractions (Table 5). Only the low dose of oestrogen $(1 \mu \mathrm{g})$ reduced the percentage of dienoic acids significantly (versus ovariectomized: $P<0.05$ ) but other hormone therapy had no comparable effect. Oestrogen treatment either alone or in combination, had virtually no influence on trienoic acids. On the other hand, oestrogen therapy lowered the percentage of tetra-, penta- and hexaenoic acids consistently (versus ovariectomized: $P<0 \cdot 05$ ). But combined hormone therapy was similarly effective only in the case of the penta- and hexaenoic fractions (versus ovariectomized $P<0 \cdot 05$ ).

Ovariectomy did not alter the percentage of nitrogenous bases of phosphoglycerides (versus di-oestrus: $P<0.03$, Table 6 ). Oestrogen therapy reduced the 
choline fraction (versus ovariectomized: $P<0.05$ ) but increased the percentage of serine (versus ovariectomized: $P<0 \cdot 05$ ). Combined administration of oestrogen and progesterone had no effect on these bases. The percentage of ethanolamine remained virtually unchanged after hormone administration (Table 6).

\section{DISGUSSION}

The results of this study show that the cyclic changes in the uterus associated with the oestrous phenomena are characterized by wide shifts in metabolism of lipids. The most conspicuous change is an increase in concentration of phospholipids during oestrus. This is abolished by ovariectomy but restored by oestrogen therapy. These facts suggest that such increase in phospholipids during oestrus is engineered primarily by the ovarian oestrogens. This is further indicated by the fact that the therapeutic restoration of phospholipid concentration in the ovariectomized animals is clearly antagonized by progesterone. The antagonistic effect of the latter on the action of oestrogens on the uterus is now satisfactorily established (Velardo, 1958). The reduction in uterine phospholipids during the metoestrus is also a significant pointer in this direction.

The uterine hyperphospholipidaemia during oestrus is associated with an equally marked reduction in concentration of triglycerides. The pattern appears to be similar to the one reported for the rat uterus after oestrogen treatment (Davis \& Alden, 1959). It is, however, possible that such hyperphospholipidaemia is a generalized effect of oestrogen because it is observed in some non-genital tissues as well like the serum, aorta and the liver (Denel, 1955; Aldesberg, 1959).

The increase in phospholipid synthesis is a likely manifestation of the stimulation of general anabolic processes in the uterus and of cellular growth. It is known that the phospholipids are important components of the cellular architecture and a valuable source of energy for synthesis of proteins (Thompson \& Ballou, 1956). The stimulation of protein synthesis (Hendler, 1959) and increased incorporation of radiophosphorus into uterine proteins (Davis, Meyer $\&$ McShan, 1956) under influence of oestrogens seem to support such a view.

In ovariectomized mice subjected to oestrogen therapy the uterine lipid patterns are comparable to pro-oestrus and oestrus except that the concentration of triglycerides is somewhat higher. Administration of oestrogen plus progesterone, however, reveals a pattern comparable to metoestrus. A close relationship between progesterone and epithelial basal fat has been reported in oestrogen-primed rabbits and guinea-pigs (Gilbert, 1942; Page et al., 1951).

The increased synthesis of some nitrogenous bases of phospholipids in ovariectomized mice subjected to oestrogen therapy is consistent with the observations of Herrannen \& Mueller (1956). Serine is perhaps the base to be synthesized first; ethanolamine and choline are derived from it subsequently (Arnstein, 1951). The accelerated rate of utilization of formate in the 1-G unit pool consequent upon the increased reductive potential induced by oestrogen (Herrannen \& Mueller, 1956), perhaps explains the increased percentage of phospholipid serine in the present study. Choline is derived from serine subsequently by utilizing the 1-C unit pool for two of its methyl groups, the third one being derived from the active methylation pool (Challenger, 1951). 
The increased reductive potential tends also to explain the stimulation of reductive synthesis of fatty acids in the uterus promoted by oestrogen. The latter evidently facilitates incorporation of these fatty acids into phospholipids, as there is a simultaneous stimulation of phosphotidyl serine, choline and ethanolamine synthesis. Under the influence of progesterone the synthesized fatty acids are perhaps channelled towards triglycerides for incorporation because the phospholipid bases are not available in adequate amounts.

The decrease in iodine value wherever it occurred after oestrogen therapy, is perhaps due to a dilution of unsaturated fatty acids by increased amounts of fatty acids synthesized under influence of oestrogen. It is unlikely that the latter exerts any significant influence on metabolism of unsaturated fatty acids per se. The concentration of linoleic, linolenic and arachidonic acids (which are essential fatty acids) was probably regulated by dietary fats under present experimental conditions. The slightly decreased concentration can be interpreted on the basis of dilution effect. The poly-unsaturated fatty acids of cholesteryl esters with even numbers of double bonds like tetra- and hexaenoic acids, however, show some increase for which no ready explanation can be offered at present, except that cholesterol is preferentially esterified with these fatty acids, particularly arachidonic acid, during the process of its mobilization and utilization (Sinclair, 1956).

\section{REFERENCES}

Alden, R. H. (1947) Implantation of the rat egg. II. Alterations in esmophilic epithelial lipids of the rat uterus under normal and experimental conditions. Anat. Rec. 97, 1.

Aldesberg, D. (1959) Hormonal influence on serum lipids. Amer. F. Med. 23, 769.

ARnstein, H. (1951) The biosynthesis of choline methyl groups. Biochem. J. 48, 27.

Bourg, R. (1929) Un test nouveau des modifications spontanées et provoquées de l'estrous chez la souris. C.R. Soc. Biol., Paris, 102, 592.

Burgos, M. H. \& WisLockI, G. B. (1956) Cyclical changes in the guinea-pig uterus, cervix, vagina and sexual skin investigated by histological and histochemical means. Endocrinology, 59, 93.

Challenger, F. (1951) Biological methylation. Advanc. Enzymol. 12, 429.

Davis, J. S. \& Alden, R. H. (1959) Hormonal influence on lipid metabolism of rat uterus. Anat. Rec. $134,725$.

Davis, J. S., MeYer, R. K. \& McShan, W. H. (1956) The effects of aminopterin and estrogen on the phosphate metabolism of the rat uterus. Endocrinology, 59, 505.

Denel, H. J., JR. (1955) The lipids. Their chemistry and biochemistry, vol. 2. Interscience, New York.

Dittmer, J. C., Feminella, J. L. \& Hanahan, D. J. (1958) A study of the quantitative estimation of ethanolamine and serine as phospholipids. F. biol. Chem. 233, 862.

GILBERT, C. (1942) The distribution of fat in the endometrium of the castrate rabbit after treatment with estradiol benzoate and progesterone. Endocrinology, 30, 773.

Goswami, A. \& Sadhu, D. P. (1961) Distribution of unsaturated fatty acids in pyridoxine deficient hypercholesterolemia. Biochem. 7. 78, 732.

Hendler, R. W. (1959) Passage of radioactive aminoacids through non-protein fractions of hen oviduct during incorporation into proteins. F. brol. Chem. 234, 1466.

Herrannen, A. \& Mueller (1956) Effect of estradiol on the metabolism of serine-3-C ${ }^{14}$ in surviving uterine segments. F. bool. Chem., 223, 309.

Leathem, J. H. (1959) Some biochemical aspects of the uterus. Ann. N.Y. Acad. Sci. 75, 463.

Page, E. W., Glendening, M. B. \& Parkinson, D. (1951) Cyclic biochemical changes in the human endometrium with special reference to the fibrinolytic enzyme. Amer. 7. Obst. Gynec. 62, 1100.

Sinclair, H. M. (1956) Deficiency of fatty acids and atherosclerosis. Lancet, i, 381.

Thompson, R. G. \& Ballov, J. E. (1956) Studies on metabolic turnovers with tritium as a tracer. V. The predominantly non-dynamic state of body constituents in the rat. F. biol. Chem. 223, 795.

VAN BEers, G., DE Jongh, J. \& Bolding, J. (1958) Isolation of phospholipids by dialysis through a rubber membrane. Essential Fatty Acids. Ed. H. M. Sinclair. Butterworths, London.

Velardo, J. T. (1958) The endocrinology of reproduction. Oxford University Press, New York.

Wheeldon, L. W. \& Collins, F. D. (1958) Studies on phospholipids. II. Determination of choline. Biochem. 7. 70, 43. 